\title{
Capartogramma crucicula (Grunow ex Cleve) Ross, primer registro del género para Colombia
}

\author{
Yimmy Montoya-Moreno ${ }^{1 *}$, Silvia Sala ${ }^{2}$, Amelia Vouilloud ${ }^{2}$, Néstor Aguirre ${ }^{1}$ \\ ${ }^{1}$ Facultad de Ingeniería, Universidad de Antioquia, Antioquia, Colombia. \\ ${ }^{2}$ Facultad de Ciencias Naturales, Universidad Nacional de La Plata, La Plata, Argentina. \\ *yimmymontoya3@hotmail.com
}

Recibido: 27-10-2010; Aceptado: 14-12-2010

\section{Resumen}

Objetivo. Dar a conocer el primer registro para Colombia del género Capartogramma y la especie Capartogramma crucicula (Grunow ex Cleve) Ross. Materiales y métodos. Esta diatomea es descrita a partir de muestras de epifiton colectadas durante los años 2006-2009, en la Ciénaga de Ayapel, Córdoba (Colombia). Resultados. La morfología de los materiales estudiados se describe y se mencionan algunos aspectos relacionados con su distribución geográfica y las condiciones fisicoquímicas del medio acuático en que fueron encontrados. Conclusión. Con la confirmación de la presencia de esta especie en Colombia, se amplía su distribución en Sudamérica.

Palabras clave: Carpatogramma crucicula, diatomea, perifiton, tropical, Colombia

\begin{abstract}
Capartogramma crucicula (Grunow ex Cleve) Ross, first record of the genus for Colombia. Objective. To report for the first time the occurrence in Colombia of the genus Capartogramma and the species Capartogramma crucicula (Grunow ex Cleve) Ross. Materials and methods. This diatom is described based on epiphyton samples collected during the years 2006-2009 in the Ayapel swamp in Cordoba. Results. We describe the morphology of the studied materials and present some aspects related to their geographical distribution and the physicochemical conditions of the aquatic environment where they were collected. Conclusion. The confirmation of the presence of this species in Colombia broadens its geographic distribution in South America.
\end{abstract}

Key words: Carpatogramma crucicula, diatom, tropical, periphyton, Colombia.

\section{Resumo}

Primeiro registro do gênero Capartogramma e da espécie C. crucicula (Grunow ex Cleve) Ross, para Colômbia. Objetivo. Apresentar o primeiro registro para Colômbia do gênero Capartogramma e da espécie Capartogramma crucicula (Grunow ex Cleve) Ross. Materiais e métodos. Esta diatomácea é descrita a partir de amostras de perifíton coletadas durante os anos 2006-2009, na planície inundável de Ayapel, Cordoba (Colômbia). Resultados. A morfologia dos materiais estudados é descrita e mencionam-se alguns aspectos relacionados com a sua distribuição geográfica e as condições físico-químicas do ambiente aquático em que foram encontrados. Conclusão. Com a confirmação da presença dessa espécie na Colômbia, amplia-se sua distribuição na América do Sul.

Palavras-chave: Carpatogramma crucicula, diatomácea, perifíton, tropical, Colômbia 


\section{Introducción}

El género Capartogramma Kufferath 1956 fue enmendado por Ross (1). El autor señala que el género está basado en la especie Capartogramma jeanii Kuff. proveniente del Lago Tanganyka y que éste es el mismo taxón que Hustedt en 1959 denominó Stauroneis karstenii que ya había sido mencionado, pero no válidamente publicado, como Schizostauron karstenii por O. Müller en 1910 y como Schizostauron karstenii por Zanon en 1941. A pesar de la confusión nomenclatural del taxón, según Ross (1) la descripción y las figuras de Kufferath muestran que sin duda el nombre del género se basa en esta especie. Este género es afín al género Stauroneis, aunque la región del nódulo central de Capartogramma consiste en barras silíceas que se proyectan al interior del frústulo, formando una estructura semejante a una " $X$ ". Esta estructura, el tigillum, está ubicado en la superficie interna de la valva y está conectado con el seudosepto (1) y es el carácter distintivo del género.

El género comprende unas pocas especies de distribución principalmente tropical siendo escasa la información disponible: Capartogramma crucicula (Grunow ex Cleve) Ross (1), C. pumila Metzeltin y Lange-Bertalot (2), $C$. allorgei (Manguin) D. Metzeltin y Lange-Bertalot (3), $C$. paradisiaca Novello et al. (4), C. karstenii (Zanon) Ross (1); C. rhombicum Ross (1) y C. amphoroides Ross (1). De todas ellas, $C$. crucicula es la que presenta una distribución más amplia, se trata de una especie pantropical, con algunas menciones en regiones templado-frías. En la presente comunicación se analiza la morfología de materiales colectados en siete ciénagas y cinco caños del sistema de planos inundables de Ayapel y se los compara con los de otras regiones geográficas.

La revisión de los trabajos taxonómicos publicados en Colombia (5-11) y del catálogo de microalgas colombia- nas (Duque, com. pers.) mostró que éste constituye el primer registro del género para el país.

\section{Materiales y métodos}

El sistema de planos inundables de Ayapel (Ciénaga de Ayapel) hace parte de la zona inundable de la depresión Momposina. La cuenca de la ciénaga tiene un área de $1504 \mathrm{~km}^{2}$, situada entre los 20 y $150 \mathrm{msnm}$. El territorio se ubica en la zona de bosque húmedo tropical con temperaturas entre los 26 y $29^{\circ} \mathrm{C}$, y está sometida al efecto del pulso de inundación, por lo que se presentan sectores con densas coberturas de macrófitas a lo largo del año, mientras que otras zonas exportan las macrófitas hacia el cuerpo principal o hacia los caños.

En la zona de macrófitas de varios cuerpos de agua del complejo cenagoso de Ayapel (Figura 1) se tomó una muestra de raíces de las tres macrófitas dominantes, las cuales fueron seleccionadas por inspección visual. Estas raíces fueron colectadas en el límite externo de la zona de macrófitas, se fijaron con solución de lugol (KI-I $)_{2}$ al 10\% y fueron tratadas para eliminar la materia orgánica con $\mathrm{H}_{2} \mathrm{O}_{2}$ siguiendo la norma europea (12). Las muestras para microscopia óptica (MO) fueron montadas en Naphax ${ }^{\circledR}$ y depositadas en la Colección de la División Ficología del Museo de La Plata (LPC). En la tabla 1 se presenta la información de las muestras empleadas y los números de la colección. Las observaciones se realizaron con un microscopio óptico de contraste de fases Leica DM 2500 y un microscopio electrónico Jeol JSMT 100.

La terminología seguida en la descripción es la propuesta en Ross et al. (13) y Barber y Haworth (14) y para la sinonimia se siguió a Ross (1).

Tabla 1. Material examinado

\begin{tabular}{llll}
\hline LPC & Sitio & Tipo de muestra & Fecha \\
\hline 9938 & Ciénaga de Paticos & Epifiton en Eichhornia azurea (Swartz) Kunth & $01 / 03 / 2007$ \\
9943 & Ciénaga de Ayapel & Epifiton en Paspalum repens Bergius & $01 / 09 / 2006$ \\
9944 & Ciénaga de Paticos & Epifiton en Utricularia foliosa Linneo & $01 / 08 / 2006$ \\
9953 & Ciénaga de Playa Blanca & Epifiton en Eichhornia crassipes (Martius) Solms-Laubach & $01 / 12 / 2006$ \\
9969 & Ciénaga de Ayapel & Epifiton en Eichhornia crassipes (Martius) Solms-Laubach & $01 / 01 / 2008$ \\
9972 & Quebrada Quebradona & Epifiton en Eichhornia heterosperma Alexander & $01 / 09 / 2006$ \\
9975 & Caño Barro & Epifiton en Eichhornia heterosperma Alexander & $01 / 09 / 2006$ \\
9976 & Ciénaga de La Miel & Epifiton en Eichhornia heterosperma Alexander & $01 / 08 / 2006$ \\
\hline
\end{tabular}




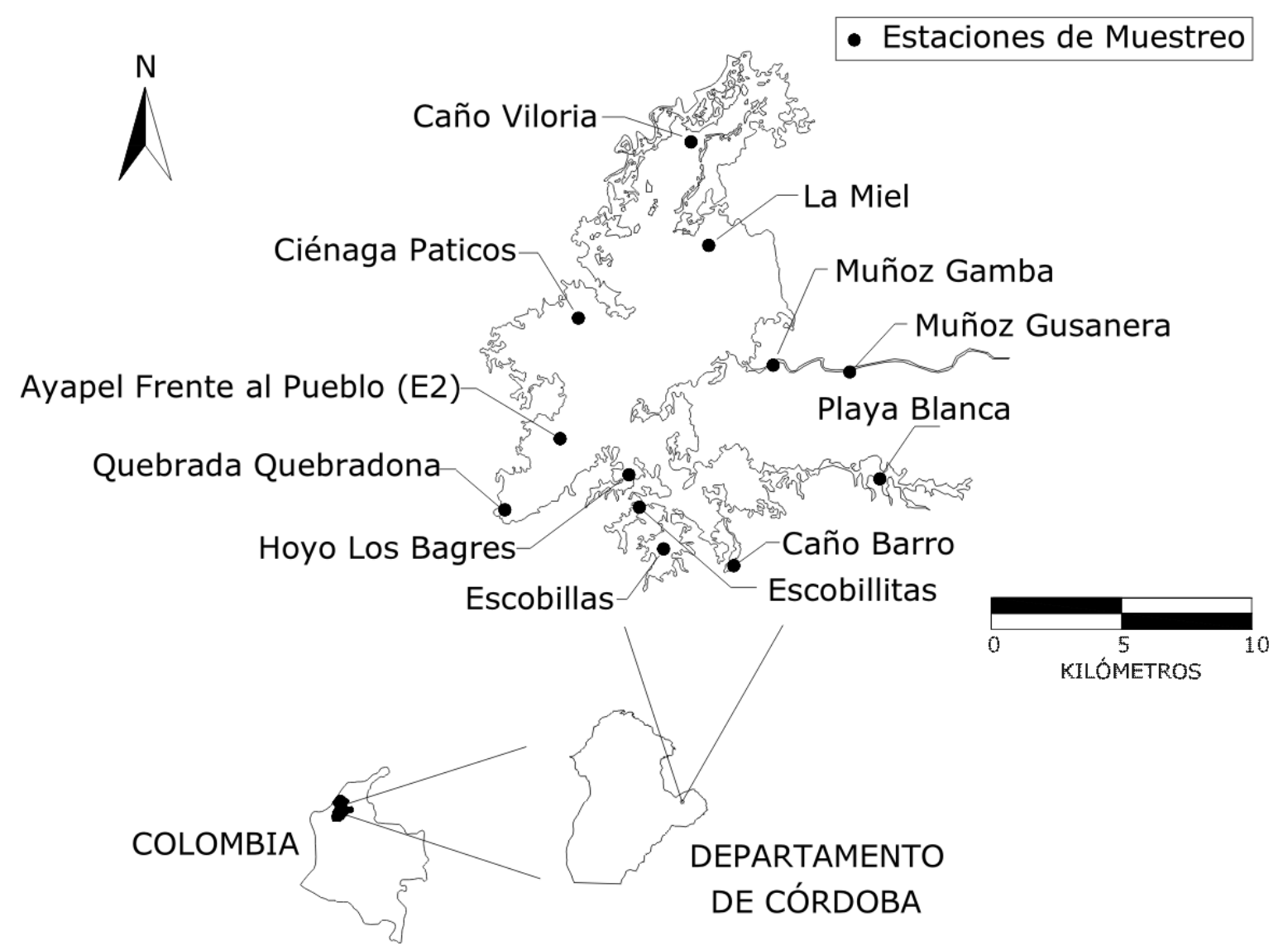

Figura 1. Ubicación de la Ciénaga de Ayapel y de las estaciones de muestreo.

\section{Resultados}

División Bacillariophyta

Class Bacillariophyceae

Order Naviculales

Family Naviculaceae

Capartogramma crucicula (Grunow ex Cleve) Ross 1963

(Figura 2a-e)

Basiónimo: Schizostauron crucicula Grun. ex. Cl. 1881

Sinónimos:

Schizostauron crucicula O. Müller 1910

Stauroneis crucicula (Grunow ex Cleve) Cleve 1894

Stauroneis crucicula (Grunow ex Cleve) Comber 1901

Stauroneis merrimacensis Woodhead y Tweed 1960

Stauroneis brasiliensis (Zimmermann) Compère 1975

Stauroneis grunowii sensu Cholnoky 1962 non Rabenhorst

Valvas lanceoladas a lanceolado-elípticas, extremos atenuado-rostrados. Rafe recto, extremos proximales expandidos en un poro, extremos distales no expandidos, levemente curvos en la misma dirección. Área central cuadrangular, prolongada internamente en una estructura en forma de " $X$ " cuyas ramas se extienden hasta los márgenes valvares denominada tigillum. Área axial estrecha y recta. Pseudoseptos en forma de arco en los ápices valvares. Las estrías transapicales son radiales en el centro de la valva, se hacen más suavemente radiales hacia los extremos y son paralelas en los ápices. Frústulo en vista conectival rectangular, ligeramente cóncavo, cingulum poco desarrollado. Las estrías y las ramas del tigillum se prolongan en el manto valvar.

Dimensiones: largo: 29-33 $\mu \mathrm{m}$; ancho: $8-9.5 \mu \mathrm{m} ; 23-28$ estrías en $10 \mu \mathrm{m} ; 40-44$ aréolas $/ 10 \mu \mathrm{m}$. Aréolas de las bandas de la cintura: 35 en $10 \mu \mathrm{m}$.

Condiciones fisicoquímicas: en el área de estudio la especie fue colectada en aguas con temperatura: $29,4-34,4^{\circ} \mathrm{C}$; oxígeno disuelto: 2,6-7,5 $\mathrm{mg} \mathrm{l}^{-1}$; $\mathrm{pH}$ : 6-7,4; conductividad eléctrica: 14,5-129,6 $\mu \mathrm{S} \mathrm{cm}^{-1}$; sólidos suspendidos: 36-76 mg ${ }^{-1}$; sólidos disueltos: $84-245 \mathrm{mg} \mathrm{l}^{-1}$; nitritos: 0,33-0,71 


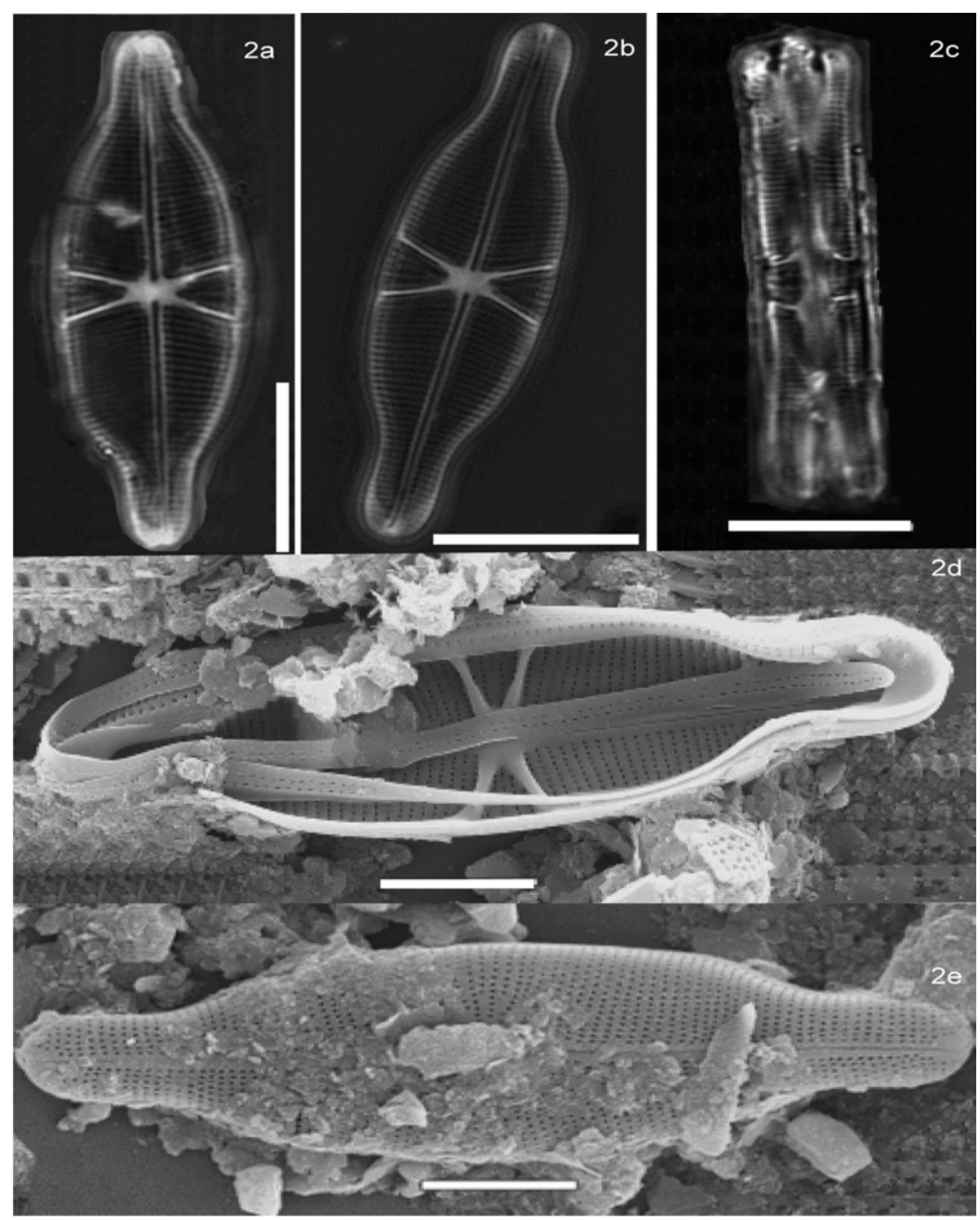

Figura 2. Capartogramma crucicula (Grunow ex Cleve) Ross. Frústulos en vista valvar (a-b) y vista conectival (c) al MO, vista interna (d) y externa (e) al MEB. Escalas=10 $\mu \mathrm{m}$ (figuras $2 \mathrm{a}-\mathrm{c})$, $5 \mu \mathrm{m}$ (figuras $2 \mathrm{~d}-\mathrm{e}$ ).

$\mathrm{mg} \mathrm{l}^{-1}$; nitratos: 0,07-5 $\mathrm{mg} \mathrm{l}^{-1}$; nitrógeno amoniacal: $0,02-$ $0,07 \mathrm{mg} \mathrm{l}^{-1}$ y sulfatos: $4-5,2 \mathrm{mg} \mathrm{l}^{-1}$.

Distribución geográfica: África (1), Argentina (15), El Salvador y Venezuela (1), Brasil, Uruguay (16), México (4, 23), USA (17,18), Australia (19), España (22), Península Ibérica (20), Francia (21-22). Brasil es el país en que se presenta mayor información sobre su distribución en diferentes cuencas $(1,24-28)$.
Abundancia relativa: Presentó valores inferiores al 1\% de las diatomeas registradas en cada cuerpo de agua en donde se reportó.

Se encontró la especie en cuerpos de agua poco profundos, con transparencia reducida, aguas cálidas, conductividades eléctricas y de sólidos en suspensión bajas y concentraciones de sólidos disueltos de rango amplio con preferencia a valores inferiores a $100 \mathrm{mg} / \mathrm{l}$. Respecto a las variables químicas, se encontró en aguas circumneu- 
trales con $\mathrm{pH}$ poco variables, en contraste, presentan características eurioicas para la concentración de oxígeno disuelto y de las formas del nitrógeno y del fósforo soluble reactivo.

\section{Discusión}

Desde el punto de vista taxonómico el estatus del género Capartogramma quedó claramente establecido en el trabajo de Ross (1) quien además hace una exhaustiva comparación del mismo con los géneros Schizostauron y Stauroneis.

De las especies que comprende el género, sólo C. crucicula presenta una amplia distribución mientras que las otras especies están restringidas a las localidades tipo, ubicadas en zonas tropicales. Ross (1) señala que el género Capartogramma y la especie C. crucicula están presentes en regiones templadas y tropicales de América y África subsahariana. A pesar de la amplia distribución en América, desde USA Kociolek y Spaulding (18) hasta Argentina Vouilloud (15), el género está registrado sólo en zonas bajas. En América del Sur no ha sido reportado en la región Andina $(15,24)$ y el registro más austral es a $34^{\circ} \mathrm{L} \mathrm{S}(C$. crucicula).

Recientemente, Blanco et al. (20), Coste y Ector (21) y Cellamare et al. (22) registraron esta especie en la Península Ibérica y Francia, consideran que se trata de una especie invasora de origen tropical o subtropical, que ha mostrado una rápida dispersión probablemente debida a su capacidad de aclimatación al igual que otras diatomeas de origen tropical en las aguas europeas.

La comparación de la morfología de los materiales colombianos de $C$. crucicula coincide en lo referente a las dimensiones, contorno valvar y características al MO, con las poblaciones de África descriptas por Ross (1), USA por Patrick y Reimer (17), México por Novelo et al. (4) y Metzeltin y Lange-Bertalot (2), Brasil Metzeltin y LangeBertalot (2) y Uruguay (16). Los trabajos de Ross (1) Metzeltin y Lange-Bertalot (2) y Metzeltin et al. (16) muestran la gran variabilidad del contorno valvar. Por el contrario, los ejemplares de Ayapel presentan un contorno uniforme, con los ápices bien diferenciados, similares a los de los ejemplares ilustrados para el Río Amazonas, ríos y arroyos uruguayos (16).

Las observaciones con microscopio electrónico de la especie son escasas. La morfología fina de nuestros materiales concuerdan con la de los ejemplares ilustrados al MEB por Metzeltin y Lange-Bertalot (2) en vista externa y detalle del tigillum y Metzeltin et al. (16), en vista externa. Sin embargo, es necesario un análisis de la morfología fina del material tipo, lo que permitiría comparar diferentes poblaciones particularmente aquellas de África y de zonas frías a fin de poder establecer si se trata de una única especie con amplia distribución, o de diferentes taxa que han sido erróneamente identificados. Hasta donde fue posible la comparación, los materiales de América del Sur desde Colombia a Uruguay presentan la misma morfología por lo que se consideran inequívocamente un único taxón.

En cuanto a las preferencias ecológicas de la especie, la información existente indicaría que se trata de una especie eurihalina. Ross (1) realiza un análisis detallado de las localidades en que fue colectada y señala que es característica de aguas dulces y ligeramente salobres, incluso fue hallada en depósitos pleistocénicos. La mayoría de los registros previos al trabajo de Ross (1) provienen de ríos, arroyos y lagunas, sobre Phragmites, Paspalidium, Potamogeton, Lagarosiphon, Ceratophyllum y sustratos duros (naturales o artificiales) en aguas someras y ocasionalmente en el plancton. El autor, señala que en el lago Tanganyika, C. crucicula fue frecuente en el epifiton y en menor medida en el bentos, al igual que $C$. rhombicum and $C$. amphoroides, ambas endémicas de este lago y además que el taxón también ha sido reportado en ambientes estuarinos de Venezuela, Brasil y Argentina-Uruguay aunque se trata de una especie poco común. Patrick y Reimer (17) la reportaron en aguas corrientes dulces pero señalan que las poblaciones con mayor desarrollo provienen de sectores con aguas salobres y Kociolek y Spaulding (18) en aguas corrientes con conductividad variable.

En Argentina y Brasil la mayoría de los registros son de agua dulce, sobre todo en ríos y arroyos (Amazonas, Iguazú); en Méjico en el perifiton y metafiton de lagunas y sabanas inundables; en el lago Michigan, en bahías poco profundas y eutrofizadas (30). Por otra parte, Moro y Fürstenberger (24) señalan que según su tolerancia a la salinidad es catalogada como eurihalina, según el $\mathrm{pH}$, esta diatomea se ha encontrado en ambientes neutros y se considera indiferente a esta variable. De Souza-Mosimann et al. (31) la registraron en contenido estomacal de peces de un lago de la Amazonia brasilera. Coste y Ector (21) la mencionan como una especie termófila que está bien representada en cursos de agua lóticos poco mineralizados y ácidos. Estos autores la citan en aguas con conductividad de hasta de $560 \mu \mathrm{S} / \mathrm{cm}$, sobre macrófitas. 
En el complejo Cenagoso de Ayapel, C. crucicula aparece como un componente temporal y de baja abundancia del epifiton de raíces de Utricularia, Paspalum repens y tres especies de Eichhornia, en varias ciénagas y arroyos, en aguas de diferentes condiciones tróficas, las cuales presentan baja profundidad, transparencia y mineralización, una temperatura del agua alta y un rango de concentración de nutrientes muy amplio.

\section{Conclusiones}

La información ecológica es fragmentaria y dispar, esto sumado a las dudas acerca de la identidad de la especie, refuerza la necesidad de profundizar los estudios de género a fin de poder establecer los requerimientos ecológicos. Con este registro de la especie para Colombia, se amplía su distribución en América del Sur.

\section{Agradecimientos}

A la Universidad de Antioquia y al grupo de investigación Gaia por su apoyo económico para la realización de una pasantía en el Museo de la Universidad Nacional de la Plata-Argentina.

\section{Financiación}

Este estudio fue financiado por el convenio Universidad de Antioquia-Gaia-Universidad Nacional de Colombia, en el proyecto Grecia-Colciencias.

\section{Conflicto de intereses}

Los autores manifiestan no tener conflicto de intereses.

\section{Referencias}

1. Ross R. The Diatom Genus Capartogramma and the Identity of Schizostauron. Bulletin of the British $\mathrm{Mu}$ seum (Natural History), Botany, 1963; 3 (2): 47-92.

2. Metzeltin D, Lange-Bertalot H. Tropical diatoms of South America I. In: Lange-Bertalot. H. (Ed.). Iconographia Diatomologica 5. Koeltz Scientific Books. Germany. 1998; 695p.

3. Metzeltin D, Lange-Bertalot H. Tropical diatoms of the South America II. Iconographia Diatomologica 18: A.R.G. Gantner Verlag K.G. Koenigstein. Germany. 2007; 877p.
4. Novelo E, Tavera R, Ibarra C. 2007. Bacillariophyceae from Karstic Wetlands in México. Bibliotheeca Diatomologica Band 54. J. Crammer. Berlin Stuttgart. $136 \mathrm{p}$.

5. Vouilloud AA, Sala S, Núñez-Avellaneda M, Duque SR. Diatoms from the Colombian and Peruvian Amazon: the Genera Encyonema, Encyonopsis and Gomphonema (Cymbellales: Bacillariophyceae). Revista de Biología Tropical. 2009; 58(1): 45-62.

6. Sala S, Duque SR, Núñez-Avellaneda M, Lamaro AA. Nuevos registros de diatomeas (Bacillariophyceae) de la Amazonia Colombiana. Caldasia. 1999; 21(1): 26-37.

7. Sala S, Duque SR, Núñez-Avellaneda M, Lamaro AA. Diatoms from the Colombian Amazonia. Cryptogamie, Algologie. 2002a. 23:75-99.

8. Sala S, Duque SR, Núñez-Avellaneda M, Lamaro AA. Diatoms from the Colombian Amazon: some species of the genus Eunotia (Bacillariophyceae). Acta Amazonica. 2002b; 32(4): 589-603.

9. Sala S, Duque SR, Núñez-Avellaneda M, Vouilloud AA. Ultrastructure of the frustule of Urosolenia species from the colombian and peruvian amazon: $U$. delicatissima nov. spec., U. amazonica nov. spec. and U. braunii (Hustedt). Diatom Research. 2008a; 23: 159-169.

10. Sala S, Ramirez JJ, Plata-Diaz, Y. Diatoms from lentic and lotic systems in Antioquia, Chocó and Santander Departments in Colombia. Revista de Biología Tropical. 2008b; 56: 1159-1178.

11. Donato-Rondón, J. 2001. Fitoplancton de los lagos andinos del norte de Sudamérica (Colombia). Composición y factores de distribución. Academia Colombiana de Ciencias Exactas, Físicas y Naturales. Colección Jorge Álvarez Lleras No. 19. 232 p.

12. CEN/TC 230. Water quality-Guidance standard for the routine sampling and pretreatment of benthic diatoms from rivers. European Standard.

13. Ross R, Cox EJ, Karayeva DG, Mann DG, Paddock TB, Simonsen R, Sims PA. An emended terminology for the siliceous components of the diatom cell. Nova Hedwigia. 1979; 64: 513-531.

14. Barber HG, Haworth EY. A guide to the morphology of the diatom frustule with a key to the british freshwater genera. Kendal: Freshwater Biological Association. (Scientific Publication, 44). 1981; 112 p.

15. Vouilloud AA. Catálogo de diatomeas continentales y marinas de Argentina. La Plata: Asociación Argentina de Ficología. 2003; 308p. 
16. Metzeltin D, Lange-Bertalot H, García-Rodríguez, F. Diatoms of Uruguay. Iconographia Diatomologica 15: A.R.G. Gantner Verlag K.G. Koenigstein. Germany. 2005; 695p.

17. Patrick R, Reimer WC. The diatoms of United States. Philadelphia, vol 1. Monogr. Acad. Nat. Sci., Philadelphia, USA. 1966; 688p.

18. Kociolek JP, Spaulding SA. Symmetrical Naviculoid Diatoms. In JD Wehr y RG. Sheath (eds). Freshwater Algae of North America. Elsevier Science, San DiegoUSA. 2003; 918p.

19. Cooper VC. Recent name changes in eukaryotic freshwater algae of New Zealand. New Zealand Journal of Botany. 2001; 39: 601-616.

20. Blanco S, Ector L, Bécares E. Diatomeas exóticas de los ríos de la cuenca del Duero. $2^{\circ}$ Congreso nacional sobre especies exóticas invasoras, León España. 2006; $71 \mathrm{p}$.

21. Coste M, Ector L. Diatomées invasives exotiques ou rares en France: principales observations effectuées au cours des dernières décennies. Systematics and Geography of Plants. 2000; 70: 373-400.

22. Cellamare M, Leitão M, Coste M, Dutartre A. Haury J. Tropical phytoplankton taxa in Aquitaine lakes (France). Hidrobiologia. 2010; 639: 129-145.

23. Figeroa-Torres MG. Diatoms in the fish rearing center in Temascal, Oaxaca, Mexico. Hidrobiológica. 2001; 11(1):85-96.

24. Moro RS, Fürstenberger CB. Catálogo dos principais parámetros ecológicos de diatomáceas não marinhas. Universidade Estadual de Ponta Grossa. 1997; 282p.
25. Lyra LT. Observações hidrobiológicas sobre a poluição do Rio Capibaribe-Mirim, Pernambuco, Brasil. Memórias do Instituto Oswaldo Cruz. 1976; 74(3/4): 361-397.

26. Torgan LC, Biancamano MI. Catálogo das diatomáceas (Bacillariophyceae) referidas para o Estado do Rio Grande do Sul, Brasil, no período de 1973 a 1990. Caderno de pesquisa - Série botânica. 1991; 3:1-201.

27. Contim LF. Contribuição ao Estudo das Diatomáceas (Chrysophyta, Bacillariophyceae) na Região da Barragem de Captação de água do Rio Iguaçu (SANEPAR), em Curitiba, Estado do Paraná, Brasil. Estudos de Biologia. 1990; 24:5-95.

28. Fonseca de Souza V. Distribuição de alguns gêneros de diatomáceas (Bacillariophyta) no saco do Laranjal, Pelotas. Monografia de conclusão de curso. Universidade Federal de Pelotas. 2005; 42p.

29. Rumrich U, Lange-Bertalot H, Rumrich M. Diatomeen der Anden von Venezuela bis Patagonien/Feuerland Und zwei weitere Beiträge. Iconographia Diatomologica 9. A.R.G. Gantner Verlag K.G., Germany. 2000; $673 \mathrm{pp}$.

30. Stoermer EF, Yang JJ. 1969. Plankton Diatom Assemblages in Lake Michigan. Great Lakes. Research Division, Institute of Science and Technology, University of Michigan, Ann Arbor, MI, GLRD Special Report No. 47; 268 pp.

31. Souza-Mosimann RM, Tavares AS. Freitas V.P. Contribuiçâo ao conhecimento da diatomoflórula do conteúdo estomacal de algumas espécies de peixes da Amazônia.I. Myleus sp. (pacu) do lago do Prato, AM., Brasil. Acta Amazonica. 1997; 27(1): 9-26. 